\title{
Médiations composites de connaissances hétérogènes : entre écrans de papier et manuscrits informatisés
}

Composite mediations of heterogeneous knowledge: between paper screens and computerized manuscripts

Philippe Marrast et Anne Mayère

\section{OpenEdition Journals}

Édition électronique

URL : http://journals.openedition.org/communicationorganisation/5183

DOI : 10.4000/communicationorganisation.5183

ISSN : 1775-3546

\section{Éditeur}

Presses universitaires de Bordeaux

\section{Édition imprimée}

Date de publication : 1 juin 2016

ISBN : 979-10-300-0059-7

ISSN : $1168-5549$

Référence électronique

Philippe Marrast et Anne Mayère, « Médiations composites de connaissances hétérogènes : entre écrans de papier et manuscrits informatisés », Communication et organisation [En ligne], 49 | 2016, mis en ligne le 01 juin 2019, consulté le 04 janvier 2020. URL : http://journals.openedition.org/ communicationorganisation/5183 ; DOI : 10.4000/communicationorganisation.5183 


\title{
Médiations composites de connaissances hétérogènes: entre écrans de papier et manuscrits informatisés
}

\author{
Philippe Marrost' ${ }^{1}$, Anne Maỵère ${ }^{2}$
}

\section{Introduction}

La production de connaissances est un processus itératif en continuel travail. Les approches en sciences de gestion ont insisté sur ces boucles d'apprentissage (Nonaka et Toyama 2003), sur cette continuité du travail de production de connaissances "dans les cadres» prescrits; d'autres travaux plus critiques ont mis en avant le travail «sur les cadres", sur ce qui fait connaissance et sur ses modes d'élaboration et de mobilisation (Bonnet et al. 2010). Dans les organisations contemporaines, cette production est de plus en plus équipée par des systèmes informatisés. Ces systèmes sont censés à la fois planifier, coordonner et tracer l'activité au quotidien, mais aussi assurer au fil de ces procéduralisations, une «capitalisation» de connaissances-clé pour l'entreprise. Au-delà de la seule organisation concernée, dans nombre de domaines, et en particulier dans le domaine de la santé ici étudié, la formalisation de connaissances prend la forme de référentiels, de procédures, et d'autres éléments de savoirs institutionnalisés, qui équipent les façons de penser et de traiter les situations.

À cet équipement est associé un imaginaire qui ferait de ces ensembles techniques, de ces savoirs référencés, les fondements d'un processus capable de s'auto-entretenir, "d'apprendre» par la mémorisation des traces de cheminement. Une critique qui peut être faite à de telles approches de «lorganisation apprenante» tient à ce qu'elles sont désincarnées et dématérialisées, et souvent fort éloignées de ce qui s'observe en situation.

Nous proposons de partir de la matérialité des objets et des pratiques pour considérer ce en quoi ils contribuent à produire des formes de savoirs situés

1 Philippe Marrast est enseignant agrégé, docteur en sciences informatiques à l'Université Toulouse 3, qualifié en $71^{\mathrm{e}}$ et $27^{\mathrm{e}}$ sections. Ses recherches portent sur les dynamiques entre pratiques d'écriture, organisation de l'activité et équipement dans la santé ; philippe.marrast@iut-tlse3.fr

2 Anne Mayère est professeure en sciences de l'information et de la communication, Université Toulouse 3, CERTOP UMR CNRS 5044. Ses recherches portent sur la rationalisation des activités de production d'information et de communication ; anne.mayere@iut-tlse3.fr 
qui servent d'appui, et qui sont produits dans et pour l'activité, de façon à mieux en cerner la diversité, la dynamique et les tensions qui les traversent et les transforment. Il s'agit de prendre en compte les sociomatérialités pour mieux comprendre les productions de connaissances à l'œuvre, en observant les objets, les systèmes techniques mobilisés dans les activités étudiées, et ainsi d'identifier ce en quoi ils sont objets de médiation autant qu'ils font médiation entre les connaissances. Corollairement, nous nous intéresserons aux acteurs individuels et collectifs pour comprendre ce qu'ils font des savoirs institutionnalisés et des équipements associés, mais aussi ce qu'ils font avec et sur les cadres pour rendre compatibles les divers ordres de connaissances en tensions.

\section{La montée en puissance de connaissances institutionnalisées équipées à l'hôpital}

Notre contribution s'intéresse au travail de la connaissance dans le domaine des soins en cancérologie, et place la focale plus particulièrement sur les pratiques d'écriture professionnelles des infirmières qui y contribuent. Ces écrits et ces pratiques participent de processus communicationnels complexes, pluriels et hétérogènes de création, de manipulation et de stabilisation de connaissances co-constitutives de l'organisation de l'activité et de la compréhension des situations de soins (Grosjean et Lacoste 1999; Strauss 1988). Ces connaissances sont autant le matériau que le produit de l'activité; des ressources hybrides, inscrites dans une variété d'objets eux-mêmes forgés par et pour les pratiques soignantes, et nécessaires dans l'articulation entre savoirs formels, scientifiques ou standardisés, et savoirs situés, négociés ou contingents (Le Marec et Babou 2003).

L'hôpital est un terrain de recherche et d'observation qui se caractérise par la présence de nombreuses technologies médicales, informatiques ou plus généralement professionnelles. Dans cet environnement fortement réglementé, contraint et restructuré par de nombreuses réformes (Ogien 2009), les outils de la e-santé sont porteurs et médiateurs de scripts liés à des politiques en matière de santé publique qui visent à configurer la prise en charge des patients et le pilotage des organisations de santé (Bonneville et al. 2010). Cet équipement organisationnel tous azimuts s'est accéléré nationalement à partir des années 2000. Il s'inscrit de plus en plus fortement dans une logique d'équipement informatisé de type progiciel de gestion (ERP - enterprise resource planning) dans lesquels les connaissances sont conçues comme des collections d'informations, organisées en silos fonctionnels (administratif, médical, prescription ) et en domaines de spécialité (chirurgie, oncologie, laboratoire...) (Venot et al. 2012).

Conjointement à ce travail d'équipement informatique global des organisations de santé, la pratique médicale hospitalière autant que la pratique infirmière ont été progressivement redéfinies en référence à une approche 
scientifique et quantifiée de la décision et de la prise en charge. Ces logiques basées sur l'énonciation des faits (Médecine Fondée sur les Faits) postulent en effet que la connaissance nécessaire à la prise en charge des patients consiste en l'énumération d'éléments objectifs qui caractérisent une situation donnée (ex : Classification Internationale des Maladies - CIM). Cette connaissance objectivée permet ensuite de sélectionner la bonne pratique dans un recueil de protocoles médicaux (ex : Classification Commune des Actes Médicaux - CCAM).

Ce double paradigme de l'informativité et de l'objectivation rencontre parfaitement les logiques gestionnaires, les modèles informatiques dits ontologiques $^{3}$ de la connaissance médicale, mais aussi les besoins en reporting pour le pilotage de la santé publique. Thésaurus, nomenclatures, guides de bonnes pratiques, protocoles et leur équipement par des standards informatiques sont ainsi des objets communs dans l'instrumentalisation de l'activité médicale, et dans l'institutionnalisation des connaissances.

Mais qu'en est-il lorsque l'on s'intéresse à la connaissance telle que mobilisée dans les situations de travail, dans les pratiques? Lorsque l'incertitude, la variabilité, l'urgence des situations de soin débordent les connaissances quantifiées (Grosjean and Lacoste 1999) et les scripts pour se loger dans les interstices de ce savoir «numérique»? Qu'est-ce qui permet dans ces organisations pluralistes, intrinsèquement aporétiques (Dujarier 2008) et fortement équipées, d'articuler la connaissance de la situation, la prescription de l'action et les connaissances professionnelles, scientifiques et règlementaires pour permettre l'action collective coordonnée? En quoi consiste le travail d'ajustement, de récriture, d'ingénierie et d'assemblage hétérogène, et que nous dit-il sur la production de connaissances?

\section{Méthodologie : Déplier le réseau hétérogène des pratiques d'écritures et les connaissances associées}

Cet article prend appui sur une étude de cas longitudinale approfondie, qui s'est déroulée dans un hôpital régional spécialisé de 2008 à 2014.

Après discussion avec le management des soins infirmiers, nous avons sélectionné principalement trois services de soin pour des patients atteints de cancer. Nous cherchions à obtenir un échantillon significatif de la variété des pratiques soignantes et des co-dépendances qui existent entre le type de pathologie, le type d'actes médicaux, l'activité de prise en charge des patients et la médiation informatique du travail. Nous avons ainsi été orientés vers un service d'hospitalisation de jour, un service de chirurgie et un service de soins palliatifs.

3 Une ontologie (du point de vue de l'informatique médicale) « implique ou comprend une certaine vue du monde par rapport à un domaine donné. Cette vue est souvent conçue comme un ensemble de concepts - e.g. entités, attributs, processus -, leurs définitions et leurs interrelations. On appelle cela une conceptualisation » (Gruber [1993] et M. Uschold et al. [1996] dans Charlet $2005: 321$ ). 
Plusieurs auteurs soulignent le rôle central des sociomatérialités dans la compréhension des environnements professionnels et des dynamiques sociotechniques (Bruni 2005; Le Marec et Babou 2003; Nicolini 2009; Orlikowski 2007). Les techniques d'ethnographie multimodale (Dicks et al., 2006) dont la photo et la vidéo ethnographie sont particulièrement efficaces pour rendre compte de la nature de ces sociomatérialités, de leurs agentivités dans l'activité et des dynamiques à l'œuvre dans le travail de construction de la connaissance qu'elles participent à accomplir. Ces techniques nous ont notamment permis, à travers la prise de note, l'analyse des documents produits, la photographie des environnements de travail et des scènes interactionnelles, la captation vidéo de certaines pratiques d'écriture, etc., de capter la richesse des situations et les dynamiques à l'œuvre dans la manipulation, la négociation entre soignants ou l'inscription de connaissances sur les nombreux supports de l'environnement (Borzeix and Cochoy 2008; Grosjean and Lacoste 1999; Jeanneret 2007). Ces approches permettent notamment de «revenir» dans les scènes interactionnelles et de percevoir plus tard des éléments d'analyse difficiles à appréhender au moment de lobservation ou dans les analyses préliminaires, ce d'autant plus que sont mobilisés des savoirs très spécialisés que nous avons dû progressivement expliciter.

Nous avons ainsi produit un matériau constitué de plus de 100 heures d'observations, de plus de 500 photos et plus de 10 heures de vidéos; complété par des entretiens (19) avec des médecins, ingénieurs, cadres de santé, et observé des réunions ou des séquences de formation accompagnant le déploiement de nouvelles applications (prescriptions, planification, dossier de soins).

Nous avons également analysé le cahier des charges du logiciel de soin, la notice utilisateur, le schéma du système d'information pour essayer de faire sortir de l'ombre les outils techniques et leurs infrastructures.

Le fait que cette recherche se soit déployée sur six années et dans une démarche interdisciplinaire entre SIC et sciences informatiques nous a permis de questionner de manière itérative, par explorations successives, en interrogeant cette organisation pluraliste et complexe dans son travail quotidien de création, d'actualisation et de gestion de connaissances. En suivant les différents actants présents ou présentifiés dans les échanges (Cooren et al. 2008; Vasquez et Marroquin 2008) nous avons cherché à appréhender cette organisation et ses processus organisants dans leurs différentes dimensions d'activité, de management, de pilotage et d'équipement informatique par des mouvements de zoom-in et de zoom-out (Nicolini 2009).

Nous avons utilisé complémentairement la technique d'investigation du shadowing (Bruni 2005) et de la biographie d'objet (Bonnot 2009) pour appréhender les environnements de travail, les objets et les pratiques à travers leur «socio-matérialité» et leur hétérogénéité, afin de caractériser 
les nombreuses interactions qui les créent, les relient, les chargent de valeurs diverses et les entretiennent.

Cette étude de cas a été complétée d'investigations similaires quoique moins conséquentes dans d'autres hôpitaux, et dans des réseaux en charge du développement de dossiers patients partagés. La mise en discussion de nos résultats avec ceux d'autres recherches a contribué également à assurer une forme de montée en généralité bien sûr toujours en chantier.

\section{Les connaissances institutionnalisées et leurs équipements : primat de la «liste»}

L'informatique est un élément essentiel dans le processus de modernisation du système de santé global, qui a connu un tournant gestionnaire important et des transformations majeures au niveau économique, managérial et organisationnel (Bonneville et al.2010).

La connaissance en tant que constituant central de la pratique médicale et infirmière n'a pas échappé à ces mouvements de rationalisation et d'informatisation. L'informatique médicale se propose d'équiper l'action, la décision et la capitalisation de connaissances. Elle doit elle-même se plier à des standards informatiques extrêmement rigoureux et nombreux qui imposent notamment à ces outils d'être accrédités pour pouvoir être utilisés dans les établissements.

Malgré cette maille technologique très serrée, nos observations de terrain ont montré qu'en pratique la connaissance déborde constamment de ces cadres formels. Les pratiques d'écriture participent ainsi d'un travail d'articulation entre une connaissance diffractée, plurielle, hétérogène et distribuée entre les soignants, l'informatique et des réseaux d'écrits aux matérialités nombreuses. Ce travail d'écriture et d'articulation, malgré son importance pour la connaissance des situations et la coordination de la prise en charge des patients, reste pourtant relativement invisible autant qu'invisibilisé dans les poches des soignants (Mayère et al. 2012).

Au-delà de la prise en charge des patients, une partie non négligeable du travail des soignants est communicationnelle, et relative à la production de connaissances, pour mener l'activité de court terme et l'articuler sur des apprentissages de moyen et plus long terme (Figure 1 page suivante).

Ces processus de création de connaissances se stabilisent temporairement sous la forme de textes organisationnels, de composites (Le Marec et Babou, 2003; Zacklad 2005) qui donnent une prise aux collectifs et aux individus pour organiser et réaliser les activités. Comme nous le montrerons, ces composites sont situés dans un entre-deux toujours en construction entre le savoir antérieur, le dire et le faire ; et ils sont maintenus par un important travail d'écriture professionnelle que nous allons rendre central dans notre analyse de la situation. 


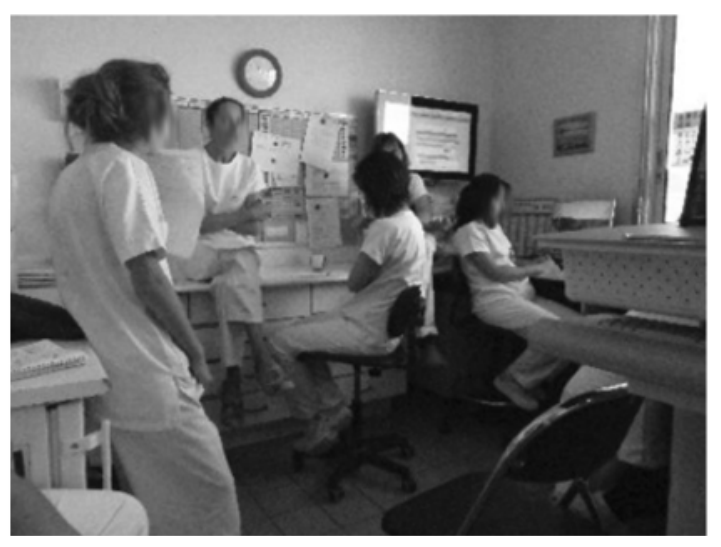

Figure 1: Les transmissions comme activité de narration, d'écoute et de débat. La concentration de chacun est intense. Ici, le réseau sociotechnique se donne à voir : entre écrans informatiques, plannings muraux, notes manuscrites, acteurs humains et pratiques de communication.

Source : auteurs

La sociomatérialité du travail d'écriture et de ces composites renvoie aussi à la notion de préhension de l'environnement, d'engagement corporel - chorégraphique- (Bruni 2005) dans la réalisation des activités. Cette sociomatérialité permet d'expliquer comment cet environnement construit par les soignants fait sens pour eux. L'environnement est acteur, notamment par sa matérialité, de la construction de l'intercompréhension collective, et de la stabilisation temporaire des connaissances.

Le logiciel de soin, le dossier médical informatisé et les différents écrans d'affichage, ne constituent qu'une composante du réseau sociotechnique qui participe de la construction de connaissances. Ces applications informatiques ne rendent disponible qu'une partie de la connaissance, et sous une forme qui n'est que partiellement adaptée à son exploitation dans le cours d'action.

L'analyse de ces composites nous a permis de mettre en évidence le travail d'ingénierie hétérogène qui permet aux acteurs de négocier et d'aligner en permanence le prescrit et le contingent pour que le travail se fasse (Grosjean et Lacoste 1999; Strauss 1988). Ce travail les amène ce faisant à construire des formes situées, très fines et ciblées de médiation entre différents ordres de connaissances.

\section{Pratique et heuristique annotatives: la dialectique liste/récit dans la production de connaissances}

La situation et la prise en charge d'un patient se trouvent à la convergence entre différentes histoires et écritures, chacune de nature et de forme particulières: entre le script de la prise en charge, l'histoire de la pathologie, l'état actuel du patient et les évolutions du cours d'action du fait des nombreuses contingences qui peuvent survenir durant l'hospitalisation.

Au fil de nos observations, durant nos interviews et nos nombreuses discussions avec les soignants, nous nous sommes intéressés à la façon dont 
ces tensions apparaissent, sont résolues puis consignées sur différentes fiches ou pense-bêtes. Comment sont-elles convoquées durant les transmissions infirmières? Sous formes d'éléments factuels (constantes, évènements) qui sont pris en notes par les soignants au cours de relèves, ou bien d'éléments à discuter et à négocier ou encore en fonction des évènements ou nouvelles informations qui occurrent durant les visites.

La communication telle que nous avons pu l'observer dans ces services est orientée vers la construction d'une connaissance partagée pour aider à la répartition, à la coordination et à la réalisation des activités de soin. Les connaissances ainsi produites et partagées permettent l'ajustement des cours d'action. Elles permettent aussi de faire collectivement sens, de mettre les situations en perspective et en regard d'expériences antérieures et de construire des projections sur l'a-venir. Les connaissances sont construites durant la négociation des différentes situations auxquelles un service doit faire face, et elles permettent d'articuler les différents systèmes d'activité.

Ainsi, afin d'appréhender la complexité des situations (pathologies patients, diverses spécialités médicales, évènements et contingences de l'activité, temporalités, ) nous avons identifié deux pratiques complémentaires auxquelles les soignants ont recours et qui s'inscrivent dans ces supports hétérogènes:

La pratique annotative qui découpe «le monde » en éléments simples de très petite taille, relativement indépendants les uns des autres, que les collectifs de soignants négocient et que l'écriture heuristique met en réseau, remet en forme et réassemble pour différents contextes professionnels et contextes d'usage (Marrast 2015).

L'écriture heuristique qui permet de créer des classifications souples et ouvertes de toutes sortes à l'aide de différents artefacts qui combinent visuellement, logiquement, spatialement, temporellement, différentes dimensions de l'activité et différentes informations relatives aux patients ou aux services ( $i b i d$. .).

Boudes et Browning dans leur travail sur les dynamiques entre textes et récits, développent l'idée que les organisations sont en tension entre deux modes argumentatifs. Le mode paradigmatique «repose sur des argumentations logiques et des modèles explicatifs étayés» et le mode narratif «se fonde sur une mise en récit qui permet de fabriquer du sens en intégrant des éléments isolés dans un tout cohérent.» (Boudès et Browning 2005).

$\mathrm{Du}$ point de vue de la médiation des savoirs sur lesquels s'appuie l'argumentation, Browning fait l'hypothèse que le mode paradigmatique s'inscrit «sous la forme de listes [...] ou bien d'items d'une procédure» qui sont dans notre cas informatisés, tandis que le mode narratif se retrouve plus particulièrement dans les récits organisationnels, les transmissions infirmières et les discussions de couloir. La fabrique de la connaissance individuelle et collective, telle que nous l'avons observée, se produit dans la résolution des 
tensions entre ces deux modes de représentation des patients et des situations qui permettent aux équipes en charge des patients de trouver la bonne distance.

Browning décrit la liste comme un objet de synthèse qui permet d'objectiver les situations et les phénomènes en réduisant le nombre de paramètres pour leur description afin de donner de la prise et du pouvoir agir, ce qui en fait un "formalisme particulièrement bien adapté à la formulation des procédures. " D'autre part, la liste possède un caractère performatif car elle "a valeur d'instruction» et qu'à travers sa " capacité de prescription, une liste est un relais de l'exercice du pouvoir» (Boudès et Browning $2005: 236$ ).

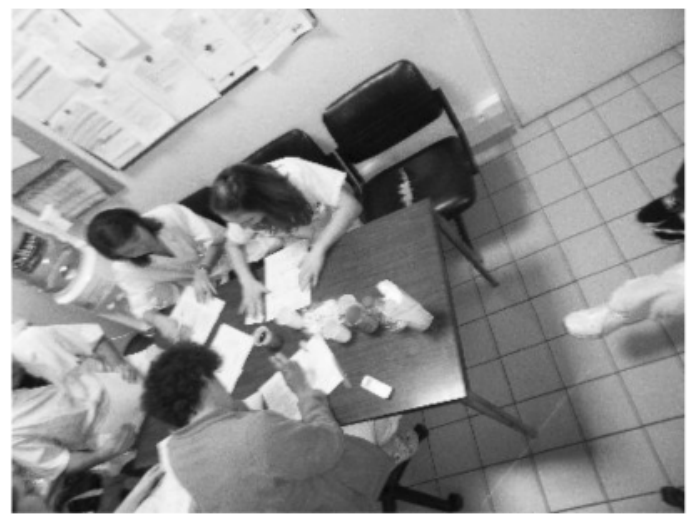

Figure 2: Transmissions infirmières entre équipe du matin et de l'aprèsmidi en présence de l'interne qui réactualise l'information des patients. Les fiches annotées sont mobilisées et mises en scène pour raconter les histoires, appuyer certains éléments narratifs et négocier l'organisation du travail à venir.

Source : auteurs

La liste est un candidat idéal pour l'informatisation ; elle produit ainsi une mise à distance des patients, des situations à travers la réduction des caractéristiques, la formalisation des procédures et son caractère prescriptif qui ignore les évolutions de l'histoire des patients.

Pour Browning le récit est une sorte de contrepoint de la liste. «Un récit permet de représenter le temps [] avec un effet de cohérence» et de relater «des événements particuliers en les intégrant dans un format universel». Les récits permettent d'articuler «la perturbation et le canonique» et de les rendre cohérents dans la narration à travers ce qu'il appelle "l'intrigue ", l'évolution de la trajectoire du patient par exemple. En redéfinissant dans l'activité «ce qu'est ou doit être le canonique, le normal, le souhaitable», le récit permet dans l'intention du narrateur de prendre une position morale particulière dans une situation donnée.

Le récit tel quon peut l'observer dans les transmissions infirmières, redonne un contexte à la liste. Il ouvre la synthèse d'un patient à la discussion et à des interprétations multiples durant les transmissions, permettant une redistribution du pouvoir de la liste entre tous les participants et créant l'arène d'une renégociation ouverte et active du canonique et de la perturbation dans le contexte particulier du récit (Figure 2).

L'enjeu du travail de ces petites mains de la connaissance réside dans l'articulation des savoirs hétérogènes nécessaires à la prise en charge des 
patients. Les savoirs professionnels médicaux et infirmiers, les perspectives gestionnaires, économiques ou parfois juridiques doivent être combinés dans l'activité à des informations plus situées concernant les patients, la situation du service, les différentes temporalités,...

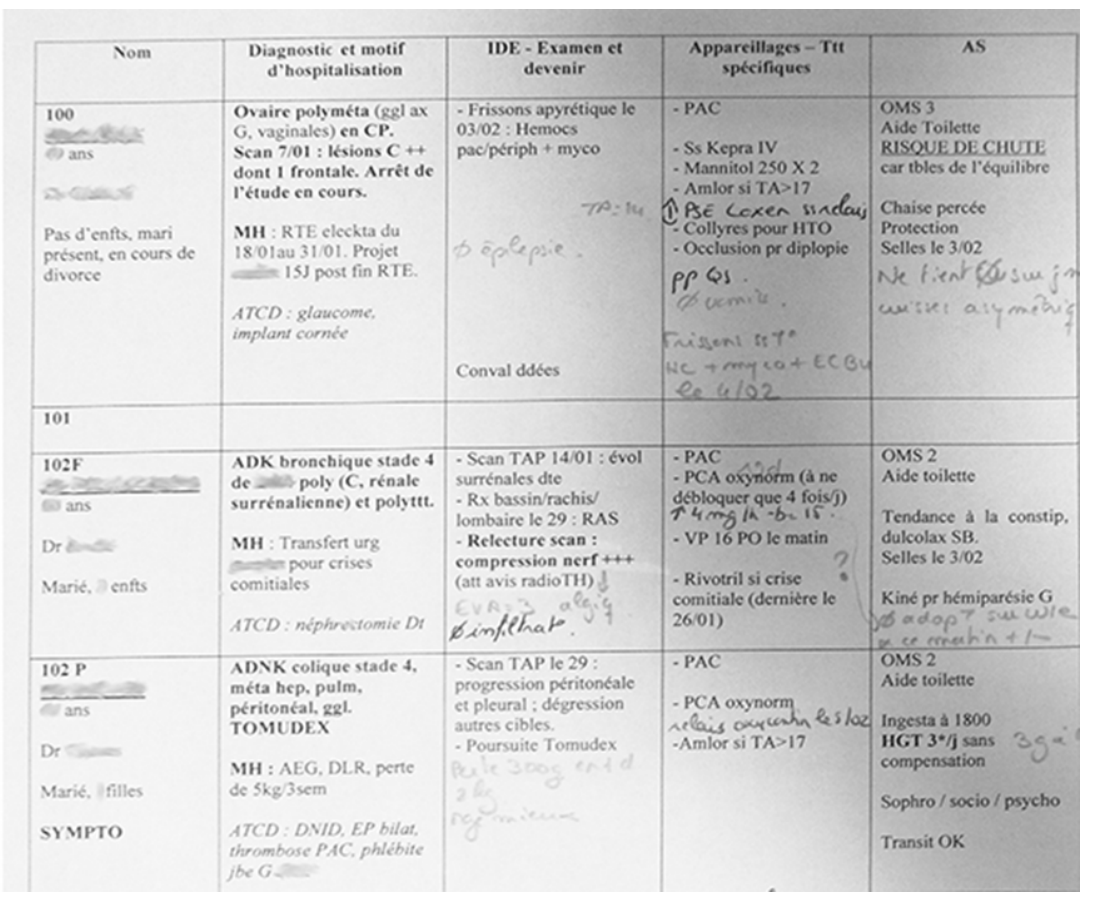

Figure 3: Fiche de transmission et de suivi des patients d'un couloir en soins palliatifs. L'écriture est à la fois synthétique, organisée en liste, libre, annotative, ouverte à différentes vues et utilisable pour l'organisation de l'action collective et les transmissions.

Source : auteurs

Ces pratiques d'annotation et d'écriture heuristiques sont de notre point de vue des outils autant que des pratiques développés par les soignants qui leur permettent d'adresser à la fois le mode paradigmatique et le mode narratif de la connaissance tout en contrôlant leur distance aux situations: physique (l'annotation et la matérialité), morale ('organisation heuristique de la connaissance) et narrative (les transmissions). Ces pratiques-outils permettent des vues synthétiques, collectives et partagées, autorisent l'écriture individuelle sous la forme d'annotation de fiches, et sont utilisées pour les récits et les négociations puisque les documents sont mobilisés lors des transmissions de relève d'équipe durant lesquelles chaque item est activement discuté par le collectif. 
Le document (Figure 3) par exemple, est un support des écritures collectives et individuelles qui reste très souple d'emploi et ouvert aux bricolages et aux appropriations diverses. Les repères collectifs en colonne restent malléables et ont été conçus pour pouvoir se chevaucher, s'articuler ou se remplacer selon les situations. L'annotation des documents imprimés est libre, les catégories et la mise en page permettent de constituer un réseau visuel de concepts et de caractéristiques interreliés qui produisent une intercompréhension partagée.

Ces connaissances et leurs médiations par ces composites organisationnels (Le Marec et Babou 2003) sont intrinsèquement paradoxales, dialectiques voire controversées car elles naissent de tensions entre des logiques locales de customisation, d'utilisation flexible et située, et des logiques externes de standards, d'intelligibilité et de continuité. Elles sont à la fois ouvertes et fermées car elles opèrent à l'intérieur et à l'extérieur de collectifs et de pratiques organisationnelles et nécessitent l'engagement des acteurs dans leur création, leur maintien et leurs adaptations.

Ces composites qui articulent les connaissances plus ou moins étroitement, deviennent des infrastructures collectives dans la répétition, l'actualisation, la négociation et grâce aux relations qu'ils entretiennent avec les pratiques qui les façonnent. Lorsque les compromis entre l'ouverture et la fermeture, entre la malléabilité et la stabilité, entre les logiques locales et globales se réalisent, l'infrastructure se produit, la technique de production de la connaissance se concrétise (Feenberg 2004; Orlikowski 2007).

\section{Conclusion}

Sil'informatisation delaconnaissancehospitalières'accordeparticulièrement bien avec le mode paradigmatique et la gestion de la connaissance sous forme de listes, elle n'a pas accès au mode narratif, ni aux dynamiques de la dialectique entre listes et récits, aux nombreuses négociations et ajustements du cours d'action.

Le contexte de numérisation généralisée en médecine hospitalière, en provoquant un déséquilibre dans les pratiques organisantes, nous a donné à voir des mécanismes d'adaptation, de bricolage et de construction dans une perspective sociotechnique de la connaissance comme élément co-constitutif des pratiques et des organisations professionnelles.

D'un point de vue méthodologique, l'analyse de la sociomatérialité et de la genèse des médiations de ces connaissances par les textes composites que nous avons présentée est importante car elles nous ont renseignés sur les relations que ces éléments entretiennent entre eux et avec les soignants, et sur les pratiques de leur écriture et de leur actualisation qui sont intimement liées avec les besoins collectifs et individuels d'information, de compréhension et de connaissance.

L'observation, la caractérisation et l'analyse de ces assemblages hétérogènes nous ont ainsi permis de tracer le travail quotidien de construction et de 
maintenance des connaissances individuelles et collectives dans toutes les formes de leur matérialité et de leur matérialisation. La forme donnée aux connaissances situées, les modes d'appropriation selon les professionnels de santé ou les collectifs qui les manipulent, le processus d'incorporation et de duplication dans divers supports et la remise en scène de ces connaissances dans les pratiques professionnelles sont autant d'indicateurs des éléments que les praticiens ont besoin d'appréhender et de manipuler pour parvenir à réaliser l'activité dans ces multiples facettes.

Notre recherche montre que la tendance lourde vers une connaissance numérisée -à nombre d'états finis - est incompatible avec les nombreuses contingences des situations, avec la nécessité d'articulation de connaissances de différents registres professionnels, de différentes natures et aux différentes matérialités, et avec le besoin de re-planification permanente des activités pour absorber l'imprévu. La contribution des SIC en dialogue avec les sciences informatiques permet de mettre en évidence la nécessaire médiation entre connaissances, et l'intérêt d'équipements pluriels et évolutifs pour mener à bien ce travail sur les connaissances pour l'action et pour apprendre de l'action. Le travail invisibilisé que cette recherche a permis de caractériser l'est doublement : dans sa mise en œuvre, et dans l'ingénierie des appuis de cette mise en œuvre. Les enjeux associés à ces questions nous semblent importants tant pratiquement, du point de vue des organisations de santé et du travail de santé, que plus théoriquement, du point de vue du questionnement requis sur ce qui s'impose toujours plus comme des «boîtes noires» non questionnables.

\section{BIBLIOGRAPHIE}

BONNET J., BONNET R., RAICHVARG D., 2010, Les savoirs communicants. Dijon: Éditions universitaires de Dijon.

BONNEVILLE L., GROSJEAN S., MAYÈRE A., 2010, Rationalisation des organisations hospitalières. Presses Univ. du Mirail.

BONNOT T., 2009, « Biographies d'objets ». Dijon SD Dispon. Em.

BORZEIX A., COCHOY F., 2008, «Travail et théories de l'activité : vers des workspace studies ?» Sociol. Trav. 50, 273-286. doi:10.1016/j.soctra.2008.06.001

BOUDÈS T., BROWNING L.D., 2005, «La dialectique entre listes et récits au sein des organisations», Rev. Fr. Gest. 233-246.

BRUNI A., 2005, «Shadowing software and clinical records: On the ethnography of non-humans and heterogeneous contexts», Organization 12, 357-378.

CHARLET J., 2005, «Ingénierie des connaissances et sciences de gestion.» Entre Connaiss. Organ. Lactivité Collect. 306-329.

COOREN F., BRUMMANS B.H.J.M., CHARRIERAS D., 2008, The Coproduction of Organizational Presence: A Study of Médecins Sans Frontières in Action. Hum. Relat. 61, 1339-1370. doi:10.1177/0018726708095707 
DICKS B., SOYINKA B., COFFEY A., 2006, «Multimodal ethnography», Qual.Res.6,77-96.doi:10.1177/1468794106058876

DUJARIER M.-A., 2008, «Prendre sur soi : l'individualisation du travail d'organisation", in La précarité : une relation entre travail, organisation et santé, Le travail en débats. Octarès, pp.107-118.

FEENBERG A., 2004, Repenser la technique, vers une technologie démocratique, Paris, La Découverte.

GROSJEAN M., LACOSTE M., 1999, Communication et intelligence collective: le travail à l'hôpital. Presses universitaires de France.

JEANNERET Y., 2007, «Les semblants du papier: l'investissement des objets comme travail de la mémoire sémiotique », Commun. Lang. 153, 79-94.

LE MAREC J., BABOU I., 2003, «De l'étude des usages à une théorie des composites: objets, relations et normes en bibliothèque ». Lire Écrire Récrire Obj. Signes Prat. Médias Informatisés 233-299.

MARRAST P., 2015, Équipement informatique des Annotations et des Pratiques d'écriture professionnelles : une étude ancrée pour l'organisation des soins en cancérologie. Université Paul Sabatier-Toulouse III, Toulouse.

MAYÈRE A., BAZET I., ROUX A., 2012, «"Zéro papier” et “pense-bêtes” à l'aune de l'informatisation du dossier de soins", Rev. Anthropol. Connaiss. 6, 1, 115-139.

NICOLINI, D., 2009, Zooming in and zooming out: A package of method and theory to study work practices. Organ. Ethnogr. Stud. Complexities Everyday Life SAGE 120-138.

NONAKA I., TOYAMA R., 2003, "The knowledge-creating theory revisited: knowledge creation as a synthesizing process », Knowl. Manag. Res. 38 Pract. 1, 2-10.

OGIEN A. 2009, "L'hôpital saisi par la quantification. Une analyse de l'usage gestionnaire de la notion de qualité», Sci. Société 30-51.

ORLIKOWSKI W.J. 2007, "Sociomaterial Practices: Exploring Technology at Work.», Organ. Stud.28, 1435-1448.

STRAUSS A., 1988, «The articulation of project work: An organizational process », Sociol. Q. 29, 163-178.

VASQUEZ C., MARROQUIN L., 2008, "À l'intersection du texte et de la conversation : comment jongler avec l'effet et le processus?» Sci. Société 26-41.

VENOT A., QUANTIN C., BURGUN A., Collectif, 2012, Informatique médicale, e-Santé : Fondements et applications. Springer Verlag France, Paris; New York.

ZACKLAD M., 2005, «Processus de documentarisation dans les Documents pour l'Action (DopA): statut des annotations et technologies de la coopération associées (nouvelle version corrigée)», in «Le Numérique: Impact Sur Le Cycle de Vie Du Document Pour Une Analyse Interdisciplinaire», 13-15 Octobre 2004, Montréal.

Résumé: L'hôpital est un terrain de recherche et d'observation qui se caractérise par la montée en puissance de connaissances institutionnalisées et équipées, qui sont au cœur du processus de modernisation du système de santé national. Notre contribution 
s'intéresse au travail de la connaissance dans le domaine des soins en cancérologie, à partir de l'observation des pratiques d'écriture professionnelles des infirmières. Ces écrits et ces pratiques participent de processus communicationnels complexes, pluriels et hétérogènes de création, de manipulation et de stabilisation de connaissances co-constitutives de l'organisation de l'activité. Cette proposition prend appui sur une étude de cas longitudinale approfondie, basée sur des techniques d'ethnographie multimodale afin de rendre compte de la nature des sociomatérialités, de leur agentivité dans l'activité, et des dynamiques à l'œuvre dans le travail de construction de connaissance qu'elles participent à accomplir. La contribution des SIC en dialogue avec les sciences informatiques permet de mettre en évidence la nécessaire médiation entre connaissances, et l'intérêt d'équipements pluriels et évolutifs pour mener à bien ce travail sur les connaissances pour l'action et pour apprendre de l'action.

Mots-clés : matérialité de la connaissance, ethnographie multimodale, connaissance co-constitutive de l'organisation, connaissances hétérogènes.

Abstract: Healthcare organizations form a research field characterized by an ever-
increasing role of the institutionalized knowledge, and its equipment. Our contribution deals
with the work of knowledge in the activities of care in oncology wards, through a specific focus
on nurse writing practices. These writings and related practices are part of complex, plural
and heterogeneous communicational processes of creation, manipulation and stabilization of
knowledge that are co-constitutive of the activity organization. This proposal relies on in-
depth longitudinal case study based on multimodal ethnography methods, in order to address
the nature of socio-materialities, their agentivity, and their contribution to the work dynamics
on diverse types of knowledge. The contributions of Information and Communication Sciences
conversing with Computer Sciences enlighten the necessary mediation between heterogeneous
knowledge, and the interest of plural and evolving equipment in order to support the knowledge
workfor the action and to sustain the knowledge production resulting from practice.

Keywords: materiality of knowledge, multimodal ethnography, knowledge co-constitutive of organization; heterogeneous knowledge. 